\title{
Pseudobulbar crying induced by stimulation in the region of the subthalamic nucleus
}

\author{
M S Okun, D V Raju, B L Walter, J L Juncos, M R DeLong, K Heilman, W M McDonald, J L Vitek
}

J Neurol Neurosurg Psychiatry 2004;75:921-923. doi: 10.1136/jnnp.2003.016485

\begin{abstract}
We describe a case of pseudobulbar crying associated with deep brain stimulation (DBS) in the region of the subthalamic nucleus (STN). Patients with pseudobulbar crying show no other evidence of subjective feelings of depression such as dysphoria, anhedonia, or vegetative signs. This may be accompanied by other symptoms of pseudobulbar palsy and has been reported to occur with ischaemic or structural lesions in both cortical and subcortical regions of the brain. Although depression has been observed to result from DBS in the region of the STN, pseudobulbar crying has not been reported. A single patient who reported the symptoms of pseudobulbar crying after placement of an STN DBS was tested in the off DBS and on DBS conditions. The patient was tested using all four DBS lead contacts and the observations and results of the examiners were recorded. The Geriatric Depression Scale was used to evaluate for depression in all of the conditions. The patient exhibited pseudobulbar crying when on monopolar stimulation at all four lead contacts. The pseudobulbar crying resolved off stimulation. This case describes another type of affective change that may be associated with stimulation in the region of or within the STN. Clinicians should be aware of this potential complication, the importance of differentiating it from stimulation induced depression, and its response to a serotonin reuptake inhibitor, such as sertraline.
\end{abstract}

W e report a case of pseudobulbar crying with features of pseudobulbar palsy that was associated with subthalamic nucleus (STN) deep brain stimulation (DBS). Clinicians should be aware of this potential complication, and its response to treatment with a serotonin reuptake inhibitor.

Pseudobulbar crying is a term used for patients who cry, but show no other evidence of subjective feelings of depression such as dysphoria, anhedonia, or vegetative signs. ${ }^{1-3}$ The syndrome may be accompanied by symptoms of pseudobulbar palsy including weakness of voluntary facial movements and hyperactive jaw, facial, or gag reflexes. ${ }^{4}$ This disorder has been reported to occur with ischaemic, degenerative, or structural changes in both cortical and subcortical regions of the brain..$^{135-11}$ Although depression has been observed to result from DBS in the region of the STN, ${ }^{12}{ }^{13}$ pseudobulbar crying has not been reported.

\section{CASE REPORT}

A 46 year old woman with an 18 year history of idiopathic Parkinson's disease presented with crying episodes coincident with activation of her left STN DBS. A left pallidotomy performed eight years previously had provided significant improvement in all the major motor signs of Parkinson's disease including rigidity, bradykinesia, and gait. The lesion, although well placed was small, and over the past couple of years she noted worsening in rigidity and bradykinesia, as well as developing a gait disorder including shuffling, start hesitation, and off freezing. She had severe on-off fluctuations with peak dose dyskinesias and off dystonia. Parkinsonian symptoms were worse on the right side so an STN DBS was implanted on the left (fig 1), the side of the previous pallidotomy. She had been treated for depression two years before the DBS surgery, but had been off all antidepressant medications without symptoms of major depression for one year. Her preoperative psychiatric screening did not reveal any history of crying spells nor was there evidence of depression or anxiety (Structural Clinical Interview for DSM-III-R (SCID), major depression, recurrent, full remission; Hamilton depression total score 8; Hamilton depression aetiologic score 0 ; Hamilton anxiety total score 7; Hamilton anxiety aetiologic score 0; Geriatric Depression Scale score 1).

After the stimulator was activated, she began to complain of fluctuating motor symptoms, cycling between off and on periods, and the development of uncontrollable crying spells. On follow up, the Geriatric Depression Scale was administered in an off DBS state, and in an on stimulation state, while off all antiparkinsonian medications for 12 hours. Monopolar stimulation (leads $0,1,2$, or 3 cathodal, case anodal, $1.5 \mathrm{~V}, 90 \mu \mathrm{s}, 130 \mathrm{~Hz}$ ) led to uncontrollable crying. There was no associated change in mood as documented by the Geriatric Depression Scale scores of $1 / 30$ for each administration of the test at each lead contact. When off DBS she had no affective symptoms, and no change on the Geriatric Depression Scale. When on stimulation at any lead contact she experienced uncontrollable crying. In addition, stimulation of the distal contact (contact 0) resulted in a subjective feeling of fear and panic, as if she was somewhere she did not want to be. Stimulation more proximally and dorsal to the STN (lead 3 cathodal) resulted in anxiety and palpitations. When switching the stimulation abruptly from "off" to "on" she sometimes experienced transient visual blurring lasting only a few seconds. She had no double vision or eye pulling. While on stimulation she appeared to all examiners to have a marked flattening of her affect as well as psychomotor retardation. She exhibited exaggerated facial and gag reflexes when on stimulation. These signs improved when stimulation was discontinued. The patient felt that she had good motor benefits with DBS, and after a lead fracture requiring later replacement, the lead was replaced into the same location. Intraoperative macrostimulation of the new lead was performed (and more ventral and dorsal locations explored for new implantation sites, without evoking additional improvements or worsening), and the new lead was anchored and confirmed with postoperative imaging to

Abbreviations: DBS, deep brain stimulation; MFB, medial forebrain bundle; SNr, substantia nigra pars reticulata; SSRI, selective serotonin reuptake inhibitor; STN, subthalamic nucleus; ZI, zona incerta 

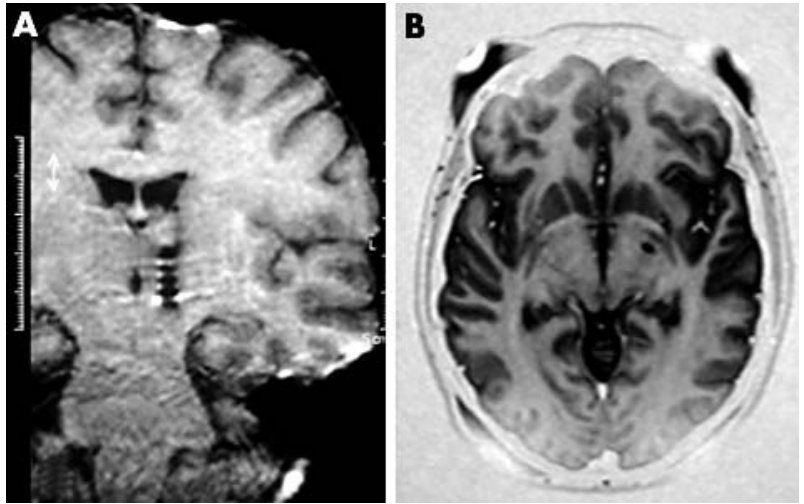

Figure 1 (A) Coronal $\mathrm{Tl}$ weighted magnetic resonance imaging scan showing the relatively medial location $(8.5 \mathrm{~mm}$ lateral) of the lead with more dorsal contacts located in the thalamus. (B) Axial image of the previous pallidotomy lesion (21.5 $\mathrm{mm}$ lateral).

be in an identical location. The intraoperative mapping and testing did not evoke any adverse mood symptoms, but did result in improvement in motor symptoms. Within 2448 hours of administration of sertraline ( $50 \mathrm{mg}$ orally) there was marked improvement of crying with DBS. Two year follow up revealed that when the patient discontinued the sertraline for one week, she experienced crying spells, fatigue, and felt "more off". After therapy was reinstituted she improved in 24-48 hours. She believed the lead continued to provide meaningful motor benefit without any "profound onoff fluctuations".

\section{DISCUSSION}

Pseudobulbar crying can be associated with lesions of the basal ganglia, thalamus, pons, subcortical white matter, and cerebral cortex. ${ }^{1}{ }^{3-11} 14-16$ Kim and Choi-Kwon in a study of post-stroke depression and emotional incontinence demonstrated that unilateral as well as bilateral lesions can cause emotional incontinence. ${ }^{5}$ Sackeim et al reviewed the reports of 119 patients who had pseudobulbar affect from unilateral lesions and found that whereas left sided lesions were associated with crying, right sided lesions were associated with laughter. ${ }^{17}$ Previous observations of DBS support this emotional response asymmetry with left sided DBS in the region of the substantia nigra pars reticulata ( $\mathrm{SNr}$ ) producing depression, ${ }^{12}{ }^{13}$ and right sided DBS in the region of the STN producing laughter. ${ }^{18}$ Our case suggests that DBS in the region of the STN can lead to pseudobulbar crying.

The reason this pseudobulbar crying occurs as a result of DBS in the region of the STN is unclear. At present the mechanisms underlying the beneficial effects of DBS are also not understood. Two potential mechanisms have been proposed. In one it is argued that DBS acts similar to a lesion. Lesions in STN have been reported coincident with emotional change. ${ }^{19}$ In our case DBS could have inhibited neuronal activity, decreasing STN output and resulting in pseudobulbar crying as a release phenomenon. The release is manifested as an affective response (abnormal outward emotion), but not a change in mood or the perception of emotion. ${ }^{4}$ An alternative hypothesis is that DBS increases output from the stimulated structure by activating fibres projecting from the site of stimulation. ${ }^{20}$ In our case this would result in activation in the medial portion (associative and limbic areas) of the STN increasing output to cognitive and mood related circuits that could also account for the present observations. This hypothesis, however, is difficult to embrace because mood was not affected in this case. In embryological development, the medial STN develops along with the hypothalamus, and the dorsolateral (sensorimotor) STN with the pallidum. ${ }^{21}$ Thus, ventral stimulation will most likely affect cognitive circuits and medial stimulation, mood related circuits. In addition, the lateral hypothalamus, the medial forebrain bundle (MFB), and zona incerta (ZI) have connections within limbic and associative circuits involved in non-motor basal ganglia thalamocortical loops and activation of these structures during DBS could also affect mood and cognitive processes. Again, it would be difficult to explain our observations on this basis because of the lack of mood change in our case. Last, the centres for facial expression have been postulated to lie in the lower brainstem, thalamus, and hypothalamus, ${ }^{22}{ }^{23}$ and therefore stimulation of any of these structures or connecting fibre systems may potentially result in pseudobulbar crying. The fact that this effect was produced during stimulation at any of the four contacts suggests that multiple different regions of the brain may be involved in mediating this effect.

One unique aspect of our case was the presence of a pallidotomy on the same side as the STN DBS. It must be considered that in some way the combination of a pallidal lesion and STN DBS may result in pseudobulbar crying. Additionally, plasticity which may lead to behavioural side effects after DBS or pallidotomy remains to be investigated further. As more groups begin to implant STN DBS in patients with prior pallidotomies these issues may become critically important.

Pseudobulbar crying can be effectively treated with tricyclic antidepressants or selective serotonin reuptake inhibitors (SSRIs). ${ }^{24-27}$ Although the mechanism for the effect of the SSRIs is unknown, it has been observed in cats that serotonergic neurones in the dorsal raphe nucleus when activated serve integrative functions in bucco-oral-facial movements. ${ }^{28}$ DBS in the region of the STN may affect the dorsal raphe and therefore serotonergic output. It has been observed that raphe neuronal activation frequently precedes the initiation of movement, stopping abruptly with pauses in the motor sequence. ${ }^{28}$ It is plausible that SSRI medications may in some way modify this midbrain serotonergic system, leading to normalisation of facial expression. Resolution of symptoms usually occurs quickly, ${ }^{16}{ }^{27}$ generally within 2448 hours with SSRI treatment. In our case of DBS induced pseudobulbar crying, we found the response to SSRI medications similar to the responses observed in patients with crying from dysfunction in subcortical and cortical structures. ${ }^{16}{ }^{24-27}$

The issue of specific mood changes at different contacts is also of great interest (table 1). The patient was fearful when stimulating ventrally (contact 0 cathodal) in the region of the SNr and anxious when stimulated dorsally in medial portions of the thalamus (contact 3 cathodal). These changes may be mediated by current spread into surrounding structures (MFB, ZI, lateral hypothalamus, brainstem) and fibre systems. Certainly, the relatively more medial location of the lead in this patient may account for some of the observed changes. In addition, her prior history of depression may

Table 1 Affective responses on and off stimulation*

\begin{tabular}{|c|c|c|c|c|c|c|}
\hline $\begin{array}{l}\text { On/off } \\
\text { stimulation }\end{array}$ & Lead & $\begin{array}{l}\text { Pseudobulbar } \\
\text { crying }\end{array}$ & Anxiety & $\begin{array}{l}\text { Depressed } \\
\text { mood }\end{array}$ & $\begin{array}{l}\text { Slurred } \\
\text { speech }\end{array}$ & Panic \\
\hline Off & Off & $(-1)$ & $(-1)$ & $(-)$ & $(-1)$ & $(-)$ \\
\hline On & 0 & $(+1)$ & $(-1)$ & $(-1)$ & $(+)$ & $(+1)$ \\
\hline On & 1 & $(+)$ & $(-)$ & $(-)$ & $(-)$ & $(-)$ \\
\hline On & 2 & $(+)$ & $(-)$ & $(-)$ & $(-)$ & $(-)$ \\
\hline On & 3 & $(+)$ & $(+)$ & $(-)$ & $(-1)$ & $(-1)$ \\
\hline
\end{tabular}

*All contacts stimulation parameters $1.5 \mathrm{~V}, 90 \mu \mathrm{s}, 130 \mathrm{~Hz}$ $(-)$, symptom not present; $(+)$, symptom present. 
provide a physiological milieu conducive to the development of changes in mood.

This case demonstrates that another type of affective change may be associated with stimulation in the region of, or within, the STN. Clinicians should be aware of this potential complication, the importance of differentiating it from stimulation induced depression, and its response to an SSRI, such as sertraline.

\section{Authors' affiliations}

M S Okun, Department of Neurology, University of Florida McKnight Brain Institute, Gainesville, FL, USA

M S Okun, Departments of Neurosurgery and Psychiatry, University of Florida McKnight Brain Institute, Gainesville, FL, USA

D V Raju, B L Walter, J L Juncos, M R DeLong, K Heilman,

W M McDonald, J L Vitek, Department of Neurology, Emory University, Atlanta, GA, USA

Competing interests: none declared

Correspondence to: M S Okun, MD, University of Florida Movement Disorders Center, McKnight Brain Institute, PO BOX 100236,

Gainesville, FL 32610 TUSA; okun@neurology.ufl.edu

Received 9 April 2003

In revised form 26 August 2003

Accepted 20 November 2003

\section{REFERENCES}

1 Brola W, Szafraniec L. [Pathologic laughing and crying in neurologic disorders]. Przegl Lek 1997;54:356-9.

2 Cantu RC, Drew JH. Pathological laughing and crying associated with a tumor ventral to the pons. Case report. J Neurosurg 1966;24:1024-6.

$3 \mathrm{Kim}$ JS. Pathological laughter and crying in unilateral stroke. Stroke 1997;28:2321.

4 Heilman K. Neglect and related syndromes. In: Heilman EVKM, ed. Clinical Neuropsychology. New York: Oxford University Press, 1979.

$5 \mathrm{Kim}$ JS, Choi-Kwon S. Poststroke depression and emotional incontinence: correlation with lesion location. Neurology 2000;54:1805-10.

6 Dark FL, MCGrath JJ, Ron MA. Pathological laughing and crying. Aust N Z J Psychiatry 1996;30:472-9.

7 Braham J. [Pathological laughter and weeping: treatment with amitriptyline]. Harefuah 1986;111:399-400.
8 Allman P. Poststroke pathological laughing and crying. Am J Psychiatry 1994; 151:291; author reply 291-2.

9 Ivan TM, Franco K. Poststroke pathological laughing and crying Am J Psychiatry 1994;151:290-1; author reply 291-2.

10 Poeck K. [Pathological laughter and crying in bulbous myotrophic lateral sclerosis]. Dtsch Med Wochenschr 1969;94:310-14.

11 Virani MJ, Jain S. Trigeminal schwannoma associated with pathological laughter and crying. Neurol India 2001;49:162-5.

12 Beijani BP, Damier P, Arnulf I, et al. Transient acute depression induced by high-frequency deep-brain stimulation. N Engl J Med 1999;340:1476-80.

13 Kumar R, Krack P, Pollak P. Transient acute depression induced by highfrequency deep-brain stimulation. N Engl J Med 1999;341:1003-4; discussion 1004

14 Yokoyama T, Imamura Y, Sugiyama K, et al. Prefrontal dysfunction following unilateral posteroventral pallidotomy in Parkinson's disease. J Neurosurg 1999;90:1005-10.

15 Okun MS, Stover NP, Subramanian T, et al. Complications of gamma knife surgery for Parkinson disease. Arch Neurol 2001;58:1995-2002.

16 Okun MS, Riestra AR, Nadeau SE. Treatment of ballism and pseudobulbar affect with sertraline. Arch Neurol 2001;58:1682-4.

17 Sackeim HA, Greenberg MS, Weiman AL, et al. Hemispheric asymmetry in the expression of positive and negative emotions. Neurologic evidence. Arch Neurol 1982:39:210-18.

18 Krack P, Kumar R, Ardouin C, et al. Mirthful laughter induced by subthalamic nucleus stimulation. Mov Disord 2001;16:867-75.

19 Martin JP. Hemichorea resulting from a local lesion of the brain (syndrome of the body of Luys). Brain 1927;50:637-51.

20 Vitek JL. Mechanisms of deep brain stimulation: excitation or inhibition. Mov Disord 2002;17(suppl 3):S69-72.

21 Groenewegan HJ, Berendse HW. Connections of the subthalamic nucleus with ventral striatopallidal parts of the basal ganglia in the rat. J Comp Neurol 1990;294:607-22.

22 Wilson SAK. Some problems in neurology. II Pathological laughing and crying. J Neurol Psychopathol 1924;16:299-333.

23 Poeck K. Pathophysiology of emotional disorders associated with brain damage. In: Vinken PJ, Bruyn GW, eds. Handbook of Neurology. New York: Elsevier, 1969.

24 Ohkawa S, Mori E, Yamadori A. [Treatment of pathological laughing with amitriptyline]. Rinsho Shinkeigaku 1989;29:1 183-5.

25 McCullagh S, Feinstein A. Treatment of pathological affect: variability of response for laughter and crying. J Neuropsychiatry Clin Neurosci 2000;12:100-2.

26 Mukand J, Kaplan M, Senno RG, Bishop DS. Pathological crying and laughing: treatment with sertraline. Arch Phys Med Rehabil 1996;77:1309-11.

27 Nahas Z, Arlinghaus KA, Kotrla KJ, Clearman RR, George MS. Rapid response of emotional incontinence to selective serotonin reuptake inhibitors. J Neuropsychiatry Clin Neurosci 1998;10:453-5.

28 Fornal CA, Metzler CW, Marrosu F, Ribiero-do-Valle LE, Jacobs BL. A subgroup of dorsal raphe serotonergic neurons in the cat is strongly activated during oral-buccal movements. Brain Res 1996;716:123-33. 